Open Access

\title{
A dataset for the development, verification, and validation of microstructure-sensitive process models for near-alpha titanium alloys
}

Adam L. Pilchak ${ }^{1 *}$, Jared Shank², Joseph C. Tucker ${ }^{2,3}$, Shesh Srivatsa ${ }^{4}$, Patrick N. Fagin ${ }^{2}$ and S. Lee Semiatin ${ }^{1}$

* Correspondence:

adam.pilchak.1@us.af.mil

${ }^{1}$ Air Force Research Laboratory, AFRL/RXCM, Wright-Patterson AFB, $\mathrm{OH}$ 45433, USA

Full list of author information is available at the end of the article

\begin{abstract}
Near-alpha titanium alloys are used for moderate-temperature applications in the early stages of the compressor in gas turbine engines. The quasi-static and fatigue properties of these alloys depend heavily on microstructure due to the absence of hard second phases and inclusions which can nucleate voids or cracks. Moreover, these alloys are known to exhibit a significant reduction in fatigue life when subjected to high mean stress or upon the application of dwell-fatigue cycles. Previous analysis has elucidated the microstructural features that drive these properties; the most important features are the volume fraction, size, and shape of clusters of similarly oriented alpha particles or microtextured regions (MTRs). To date, there have been few efforts to elucidate in a quantitative fashion the evolution of MTRs during thermomechanical processing (TMP). To meet this need, we have performed hot-compression tests on Ti-6Al-2Sn-4Zr-2Mo-0.1Si billet material with high-aspect-ratio MTRs at $0^{\circ}, 45^{\circ}$, and $90^{\circ}$ to the direction of primary metal flow during manufacture (i.e., the billet axis), thoroughly characterized the initial and final microstructures, and quantified field variables via finite-element method (FEM) process simulations for each experiment. These data can be used for a variety of purposes including the development, verification, and validation of models for microstructure/texture/microtexture evolution and defect formation.
\end{abstract}

Keywords: Titanium, Microstructure, EBSD, Texture, Microtexture, Process simulation, Characterization

\section{Introduction and background}

Near-alpha titanium alloys are an important class of materials used in demanding applications in the compressor section of gas turbine engines. These materials are typically selected because of their high strength-to-weight ratio and resistance to failure associated with creep, fatigue, and environmental degradation. They are also unique compared to other engineering materials based on $\mathrm{Ni}$, Fe, and $\mathrm{Al}$ because they have two ductile phases; lack hard second phases/inclusions, which often initiate cracks in service; and do not exhibit a ductile-to-brittle transition even at temperatures approaching $0 \mathrm{~K}$ [1]. Furthermore, these alloys exhibit a complex, hierarchical structure

(c) 2016 The Author(s). Open Access This article is distributed under the terms of the Creative Commons Attribution 4.0 International License (http://creativecommons.org/licenses/by/4.0/), which permits unrestricted use, distribution, and reproduction in any medium, provided you give appropriate credit to the original author(s) and the source, provide a link to the Creative Commons license, and indicate if changes were made. 
that necessitates characterization across orders of magnitude in length scale to capture all of the relevant features that contribute to mechanical (and physical) properties. For example, local neighborhoods and microstructural weak links drive where and when fatigue-crack nucleation occurs and how fast the early stages of growth progress while the average microstructure and global texture are more important with respect to crack propagation and fracture toughness.

When intended for rotating components in gas turbine engines, near-alpha titanium alloys are typically used in the alpha+beta-processed condition comprising a bi-modal microstructure of primary-alpha particles in a matrix of lamellar alpha+beta. The size and volume fraction of the primary alpha, the thickness of the alpha lamellae, and size of the alpha colonies (i.e., groups of parallel alpha lamellae with the same crystallographic orientation separated by thin ribs of beta phase) are the most critical microstructural features. The strong plastic (and elastic) anisotropy of the hcp alpha phase and thus the macrotexture in mill products and finished components also play an important role and can lead to variations in elastic modulus ( 30\%), yield strength ( 20\%), fatigue-crack-growth rate [2], and overall fatigue life [3]. More specifically, macrotexture, or the preferred orientation of a polycrystalline aggregate, impacts both low- and high-cycle fatigue properties; however, it has been shown that the presence and degree of microtexture is of paramount importance with regard to dwell-fatigue susceptibility [4]. Microtextured regions (MTRs) are clusters of alpha particles that appear fully spheroidized ("recrystallized") under conventional imaging modes but are found to have similar orientation when the spatial distribution of the texture components is investigated using electron backscatter diffraction (EBSD) and, in some instances, with optical microscopy [5].

Figure 1 illustrates the hierarchical nature and complexity of alpha+beta-processed titanium microstructures whose features range in size from 10s of nanometers (e.g., coherent, ordered $\mathrm{Ti}_{3} \mathrm{Al}$ precipitates) to multiple millimeters (MTRs). Because there are no hard second phases or inclusions in Ti alloys, the mechanical properties are a very strong function of the effective slip length in the microstructure. Classically, one would define the characteristic dimension to be the lath thickness, the alpha-particle size, the alpha-colony size, or some combination of these factors. However, at each length scale, there is potential for coincidence of slip systems between neighboring features of the same phase or neighboring features of different constituent phases, which would increase the effective local slip length. Consequently, the spatial distribution of the crystallographic orientation of each constituent phase is very important with respect to defining the "maximum slip length," which, when subjected to other constraints regarding orientation and neighborhood, define the size of so-called microstructural weak links. For example, Bridier et al. [6] have demonstrated slip transmission between primary-alpha and secondary-alpha colonies. Recently, Echlin et al. [7] have highlighted the development of multi-alpha-particle slip bands traversing entire MTRs. Hence, it is important to have models available to simulate microstructure evolution at each length scale. To this end, there have been significant efforts to understand and model the relationship between thermomechanical processing (TMP) parameters and microstructure in the two-phase, near-alpha, and alpha+beta titanium alloys $[8,9]$. These models have successfully predicted factors such as (i) the mean size, volume fraction, and rate of coarsening of primary-alpha particles during solution heat treatment and 


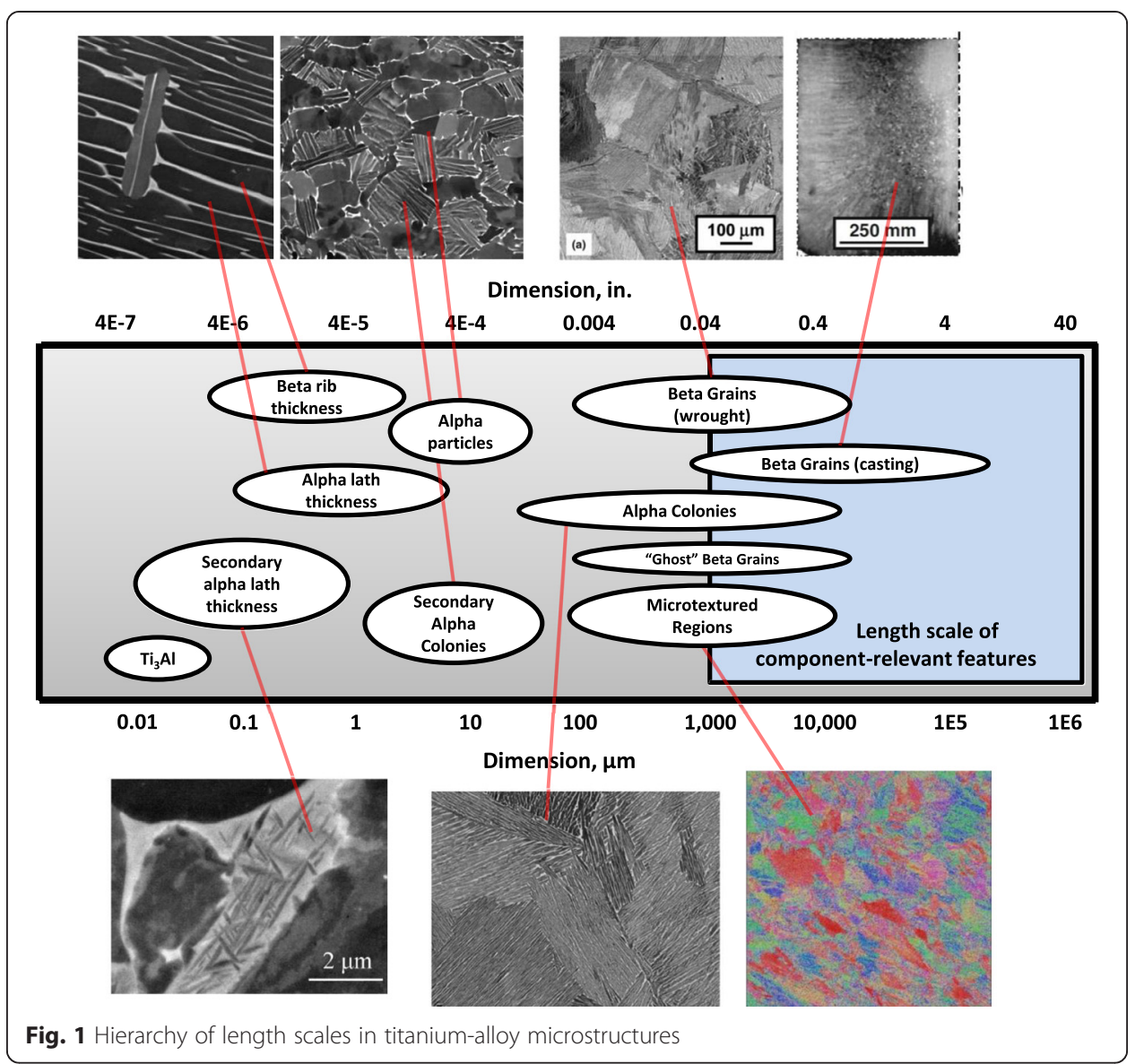

cooling, (ii) alpha- and beta-phase deformation textures, and, to a lesser extent, (iii) secondary-alpha transformation texture and lath thickness.

The various microstructural features can be used to predict key quasi-static (uniaxialtension) properties reasonably well, but the cyclic response of titanium alloys is a significantly stronger function of the hierarchical length scale of the microstructure and specifically "weak-link" microstructural neighborhoods such as MTRs. MTRs, which are the largest titanium microstructural features currently considered in the alpha+beta processed condition, can be so large that they reach design-relevant length scales (Fig. 1). For example, the thickness of the web section in a fan or compressor disk may be less than $10 \mathrm{~mm}$, resulting in relatively few MTRs per cross-section. Such a configuration leads to considerable variability in material behavior compared to bulk polycrystalline response. As a result, thin locations with respect to the average MTR size should be treated with caution in the design process, especially for low $\mathrm{K}_{\mathrm{t}}$ notches and fillets. The interaction of these stress risers with MTRs during cyclic and dwell-fatigue loading has not been investigated to any extent and thus remains a fruitful area for future research.

The potential for early crack initiation and accelerated crack growth within MTRs is of primary concern when there are few MTRs across the thickness of a part. Hence, physically large cracks can form in a small number of fatigue cycles leading to debits in total lifetime $[10,11]$. The magnitude of this debit depends on factors such as the size, shape, crystallographic/morphological orientation distribution, and misorientation 
distribution within an MTR at a particular location within a component, and the local stress state to which the part is subjected. These attributes depend on the initial distributions in semi-finished mill products and the subsequent thermomechanical history (including strain path) used to make a finished component.

To meet the need for detailed microstructure data to understand and model the evolution of MTRs (and other microstructural parameters) across the pertinent wide length scale, we provide herein a detailed dataset pertaining specifically to the TMP of Ti-6242Si. The data can also be used to develop robust segmentation and quantification routines for MTRs. Our overall intent, therefore, has been to provide information that can be used by both modelers and experimentalists alike. For the modelers, for example, we have performed an initial segmentation and quantification of MTRs based on our current internal practices. We have also provided the raw EBSD results for experimentalists who wish to perform segmentation based on their own approaches.

\section{Materials, methods, and dataset description}

\section{Availability of supporting data and citing a data descriptor article}

The datasets supporting the results of this article are available in the NIST repository, http://hdl.handle.net/11256/647 [12]. When using the results of the present work, please cite the dataset and this manuscript as they are mutually dependent on one another for meaning. The principal components of the dataset [12] include results from (i) the characterization of the microstructure of billet material on three orthogonal planes via scanning electron microscopy (SEM) and EBSD; (ii) the plastic flow behavior derived from isothermal hot-compression tests performed on Ti-6242Si at three different temperatures, three different angles to the axis of the initial cylindrical billet, and one strain rate; (iii) the finite-element method (FEM) simulations of the hot-compression tests; (iv) the characterization of the $899^{\circ} \mathrm{C}$ compression samples on three orthogonal planes with SEM and EBSD; and (v) the distributions of the MTR sizes on each plane in (iv). The above data is divided into the following subsections based on the type of information, i.e., isothermal hot compression, FEM, microscopy, and segmentation and quantification of microstructure and MTRs.

\section{Isothermal hot-compression data}

To develop an orientation- and temperature-dependent flow-stress database, isothermal hot-compression tests were performed on 25.4-mm-diameter $\times 38.1$-mm-height cylinders cut from the mid-radius position of a 209-mm-diameter Ti-6242Si billet; the composition, microstructure, texture, and microtexture of this billet have been reported previously [13]. More specifically, the samples were cut with the compression direction oriented $0^{\circ}$ (axial), $45^{\circ}$, or $90^{\circ}$ (radial) to the long axis of the billet and, by extension, to the long direction of the initially high-aspect-ratio MTRs in the billet. The samples were compressed isothermally at temperatures of $899^{\circ} \mathrm{C}, 954{ }^{\circ} \mathrm{C}$, and $982{ }^{\circ} \mathrm{C}$ on a servo-hydraulic load frame at a constant true axial strain rate of $0.01 \mathrm{~s}^{-1}$ to an average true height strain of 1.07 (i.e., a 3:1 reduction). After reaching the total reduction, each sample was water quenched (within $<1 \mathrm{~s}$ ) to retain the elevated-temperature microstructure.

The load stroke and resulting average pressure-height strain data from the compression tests were corrected for machine stiffness and die-workpiece interface friction per 
Altan [14] (assuming a friction shear factor, $m_{\mathrm{s}}$, of 0.4 ) to produce true-stress-truestrain curves. The raw and processed data are provided in a Microsoft Excel ${ }^{\mathrm{TM}}$ (Microsoft, Redmond, WA) file in which the equations are embedded so that the reader can assess the sensitivity of the friction-correction to the choice of the critical parameter, $m_{\mathrm{s}}$. The friction-corrected flow curves (Fig. 2a) revealed a strong dependence on temperature. There was also some sensitivity of the flow stress to orientation for test temperatures of $899{ }^{\circ} \mathrm{C}$ and $954{ }^{\circ} \mathrm{C}$, at which there is $\sim 75$ or $\sim 50 \%$ primary-alpha particles by volume, respectively; at both of these temperatures the axial direction was slightly stronger. At $982{ }^{\circ} \mathrm{C}$, at which the primary-alpha phase constitutes only $25 \%$ of the material by volume, most deformation is accommodated by the beta phase, which is less plastically anisotropic resulting in essentially no orientation dependence of the flow behavior, at least at the macroscopic, polycrystalline scale. The subtle differences due to orientation at the highest test temperature were within the experimental error, as shown in Fig. 2b. This figure compares data for two isothermal compression tests performed on axial samples at $954^{\circ} \mathrm{C}$. The peak stress differed by less than $2 \mathrm{MPa}$, a variation which was consistently tracked throughout the entire experiment.

\section{FEM simulations}

Two-dimensional, axisymmetric FEM simulations of the hot-compression tests were performed using the commercially available code DEFORM ${ }^{\mathrm{TM}}$ (Scientific Forming Technologies Corporation, Columbus, $\mathrm{OH}$ ) for the purpose of understanding the spatial and temporal evolution of local strain components and rigid-body rotations due to metal flow. These data were used in turn to guide the selection of locations for microstructural characterization.

The friction-corrected flow-stress data were used to develop a database that was implemented in the FEM simulations, which were run non-isothermally. In addition, the friction shear factor was tuned iteratively in the simulations until the load-stroke data and simulated cross-section shape matched the experimentally observed stiffnesscorrected load-stroke and sample cross-section results, respectively. The flow stress, friction, and various boundary conditions for the FEM code are provided as input.KEY files, which contain all of the information necessary to run the simulations inside

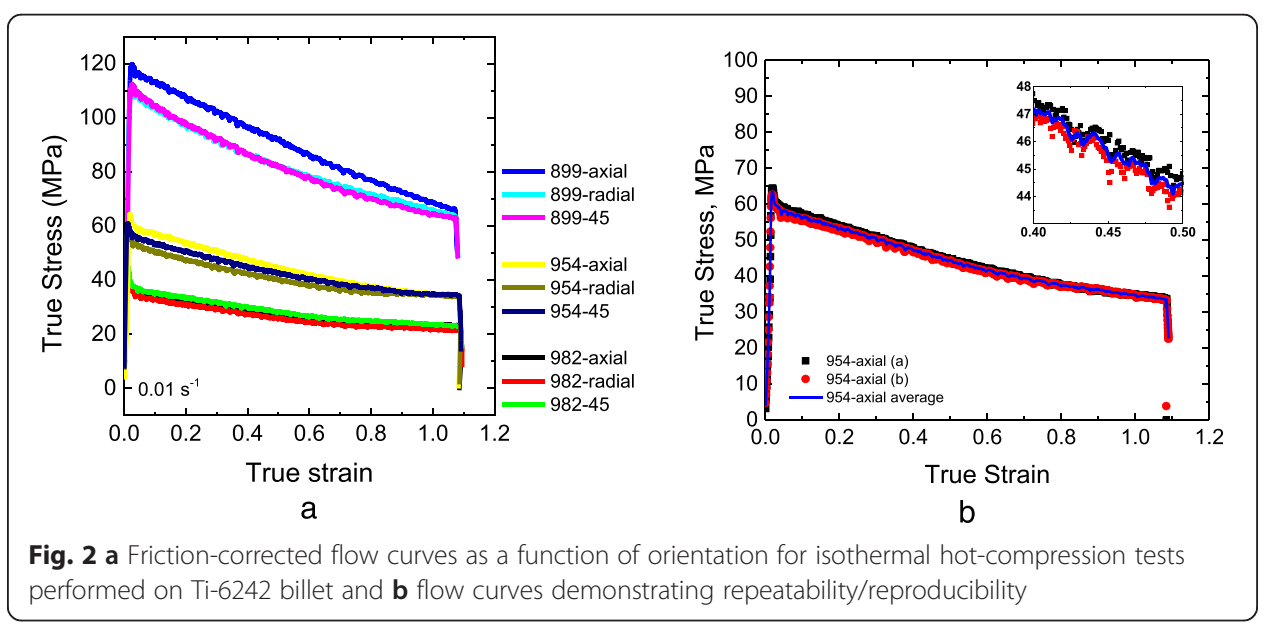


DEFORM $^{\mathrm{sx}}$. The database files (extension *.DB) are also included which contain the complete FE model. These can be loaded directly into the DEFORM ${ }^{\mathrm{mm}}$ post processor, and the thermomechanical history at each time step can be visualized. If the reader does not have access to this software, however, the other information provided in this article, including Table 1, and the corresponding supporting data are sufficient to recreate the model in any FEM package. As an example of the results from DEFORM ${ }^{\mathrm{m}}$, the initial and final sample meshes and the final effective Von Mises strain contours for the axial compression at $899^{\circ} \mathrm{C}$ are shown in Fig. 3. Simulation results for the other samples looked very similar with only very minor variations in the magnitude of local strains and hence are not shown here.

\section{Microscopy}

Based on the strain distributions predicted by the FEM simulations (with special attention to local strain gradients), a region of interest (ROI) was defined to do microstructure and texture characterization. Because MTRs can measure multiple millimeters in one or more dimensions and the size and shape of an MTR following a forming operation is a strong function of the strain path experienced during deformation, we selected ROIs near the center of each compression sample for detailed characterization. The material elements at such locations had experienced essentially axisymmetric compression with no shear strain (Table 2) and minimal rigid-body rotation. The near equality of the Von Mises effective strains and the imposed axial strain underscore the near perfect uniaxial-compression assumption. In view of sample symmetry, the ROI thus comprised a 15-mmdiameter $\times 3$-mm-height cylinder centered in the bulk of each compression sample. Due to practical limitations, however, it was only possible to perform a detailed examination on three mutually perpendicular planes within the ROI. For this purpose, each sample was sectioned via electric-discharge machining to expose three orthogonal faces. The imaging planes, the locations of EBSD analyses, and the corresponding reference frames for the billet and compression samples are shown schematically in Figs. 4 and 5, respectively. This approach provided a statistically averaged sense of the threedimensional shape of the MTRs before and after compression and the relative amount of rotation of the principal axes of the MTRs during the imposed strain. While this is a reasonable approach for modeling the average response, it does neglect the possibly important contribution of the local neighborhood to the development of intragranular strains, which may be relevant to the spheroidization process and the concomitant randomization of orientations within an individual MTR during forging. Two additional EBSD scans covering significantly larger areas are also included in the dataset. These include a scan of the full cross-section of face 1 for the $45^{\circ}$ compression sample and a scan of half the sample of the axial-compression specimen.

Table 1 Boundary conditions for the finite-element model of the hot-compression tests

\begin{tabular}{lll}
\hline Description & DEFORM variable & Value \\
\hline Heat transfer coefficient to environment & CNVCOF & $22.67 \mathrm{~W} / \mathrm{m}^{2} \mathrm{~K}$ \\
Heat transfer coefficient between workpiece and die & IHTCOF & $7360 \mathrm{~W} / \mathrm{m}^{2} \mathrm{~K}$ \\
Environment, die, and workpiece initial temperatures & REFTMP & $899^{\circ} \mathrm{C}$ \\
Die-workpiece friction coefficient & FRCFAC & 0.3 \\
\hline
\end{tabular}




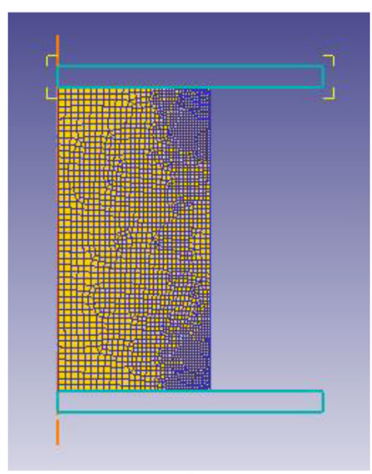

a

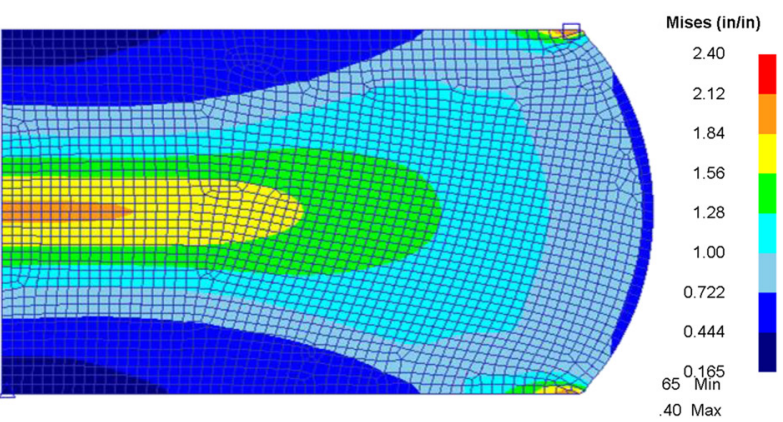

b

Fig. 3 FEM simulation of the isothermal hot-compression test: $\mathbf{a}$ undeformed condition and $\mathbf{b}$ predicted final effective strains for the axial-compression sample tested at $899^{\circ} \mathrm{C}$

Backscattered-electron micrographs were acquired on a FEI XL30 or a FEI Sirion SEM. Both SEMs were equipped with field-emission-gun (FEG) sources. To facilitate strong contrast within the microstructure, each SEM was operated at $20 \mathrm{kV}$ with a spot size of " 5 " and a 30- $\mu \mathrm{m}$ aperture producing $~ 2.5 \mathrm{nA}$ of current. Images were collected at multiple magnifications $(\times 250, \times 500, \times 1000$, and $\times 2000)$ from approximately the center of the forging in the highly compressed region of each sample. Additional $\times 500$ images were collected on each sample elsewhere within the previously defined characterization volume to assess microstructural variability. A magnification-calibration standard image collected during each SEM session is provided to facilitate accurate quantitative measurements. Representative micrographs of the as-received billet material are shown in Fig. 6 while examples from the compression samples are shown in Fig. 7. These images reveal the extent of the changes to the size, shape, and volume fraction of primary-alpha particles during heat-up, soaking for $3 \mathrm{~min}$, and the $\sim 107 \mathrm{~s}$ necessary to complete the compression test.

The extent of texture and microtexture were assessed with EBSD. These data were collected on the FEI XL30 SEM with a FEG source using a Hikari camera. Beam conditions were chosen to provide a high probe current to enhance pattern quality; for this purpose, an accelerating voltage of $20 \mathrm{kV}$ with a spot size of 5 and a $100-\mu \mathrm{m}$ final aperture were employed to produce nearly $50 \mathrm{nA}$. Using a custom batch-scan program [15], EBSD data were collected over relatively large areas using a combination of beam scans and automated stage movements. Table 3 provides a summary of the scans collected for this work and which are included in the dataset. The individual tiles in their native binary *.osc format are provided in the "RawData" folder for each sample. While there is a MATLAB (The MathWorks, Inc., Natick, MA) function that can unpack the binary files and read them into a variable in the MATLAB workspace, we

Table 2 Average values of strain components within the 15 -mm-diameter $\times 3$-mm-height "cylinder" contained within the compression sample selected for further analysis

\begin{tabular}{lcccc}
\hline Sample orientation $\left(^{\circ}\right)$ & Von Mises effective strain & Axial strain & Radial strain & Shear strain \\
\hline 0 & 1.724 & -1.706 & 0.853 & -0.003 \\
45 & 1.707 & -1.697 & 0.848 & 0.000 \\
90 & 1.710 & -1.700 & 0.850 & 0.000 \\
\hline
\end{tabular}



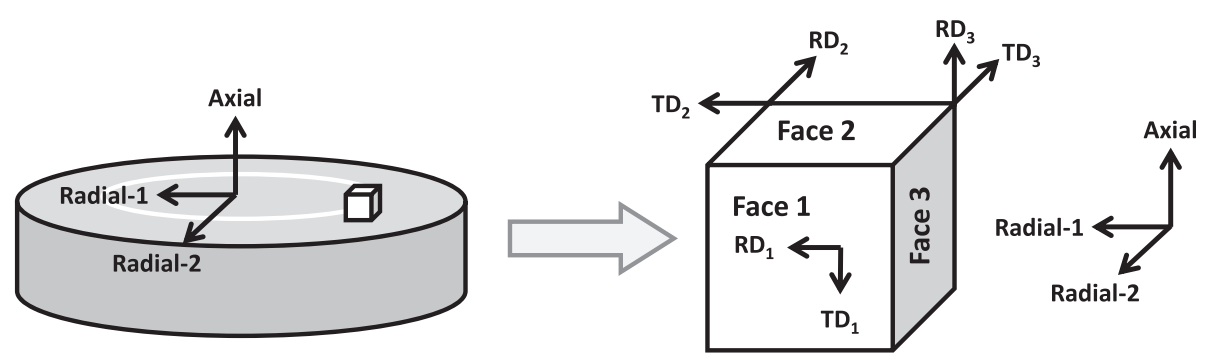

Fig. 4 Billet coordinate system and correlation to EBSD reference frames. The billet is defined by axial-radial coordinates while the EBSD scans are in the EDAX/TSL RD-TD-ND convention

have also provided the entire stitched dataset as a single ASCII file (file extension ".ang) that resulted as an output of a previously published code [16]. All of the subsequent data shown here were generated from the stitched file after having been cropped to the dimensions shown in Fig. 5b-d. The ang files are the standard ASCII format for the commercial EBSD vendor EDAX/TSL (EDAX, Mahwah, NJ). The files contain 10 columns of data that are, in order, the crystal orientation in "active" Bunge Euler angles ( $\mathrm{phi}_{1}, \mathrm{PHI}, \mathrm{phi}_{2}$ ) (following the so-called $Z-X-Z$ convention, i.e., active rotations from the "initial" sample axes to the "new" crystal axes), spatial coordinates $(x, y)$, image quality (IQ), confidence index (CI), phase identification, detector intensity, and fit. For ease of visualization, we have also provided JPEG images of the crystal
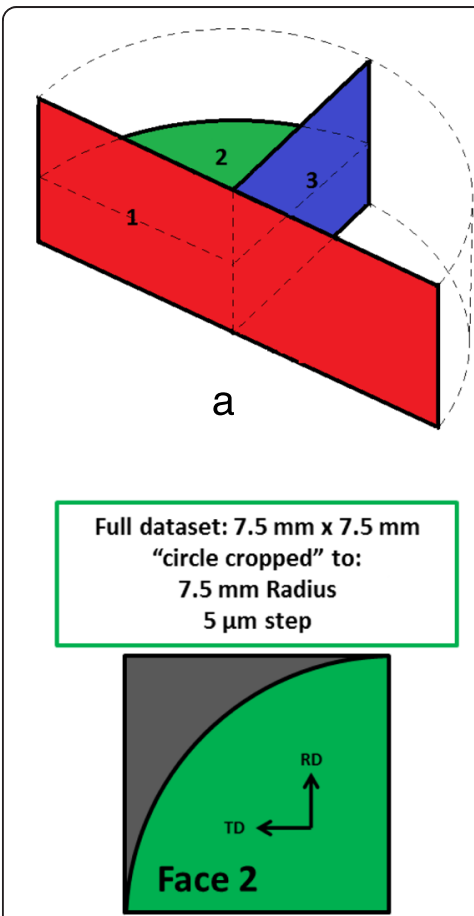

C
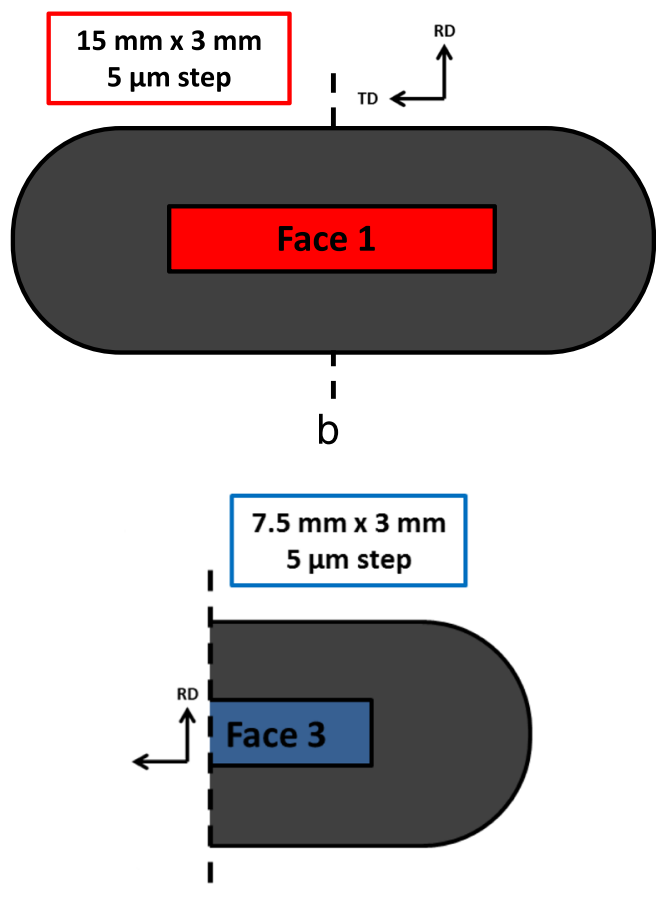

d

Fig. 5 a Schematic illustration of sectioning plan for the isothermal hot-compression samples and locations and sizes of the EBSD scans on faces $\mathbf{b} 1, \mathbf{c} 2$, and $\mathbf{d} 3$. The local coordinate system is defined by the EBSD software vendor (EDAX/TSL) and is expressed as RD-TD-ND 


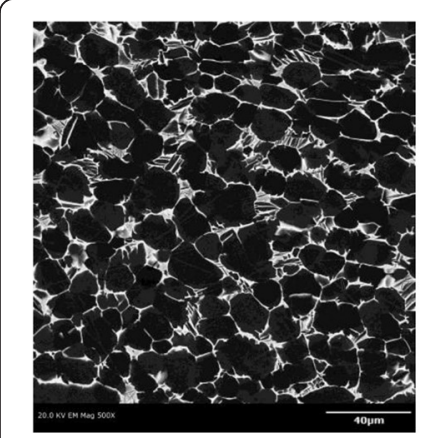

a

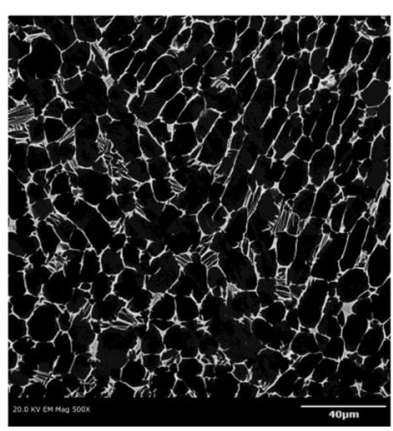

b

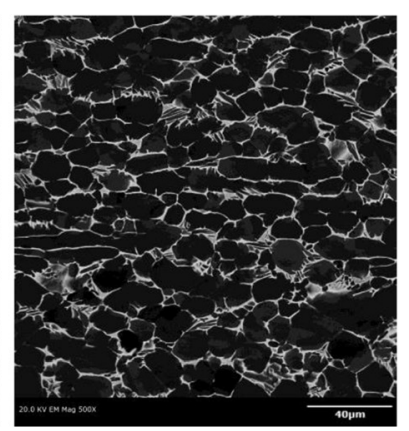

C

\section{$100 \mu \mathrm{m}$}

Fig. 6 Backscattered-electron micrographs of faces a 1, b 2, and $\mathbf{c} 3$ in the as-received billet material

orientation maps and the resulting textures derived from them for the $0001,10 \overline{1} 0$, $2 \overline{1} \overline{1} 0$, and $\overline{2} 113$ poles of the hexagonal alpha phase.

EBSD data are provided in the native reference frame, the "RD-TD-ND" coordinate system from the EDAX/TSL software, which are equivalent to the " $X-Y-Z$ " axes of a righthanded orthonormal reference system. The relationship between these axes and the EBSD

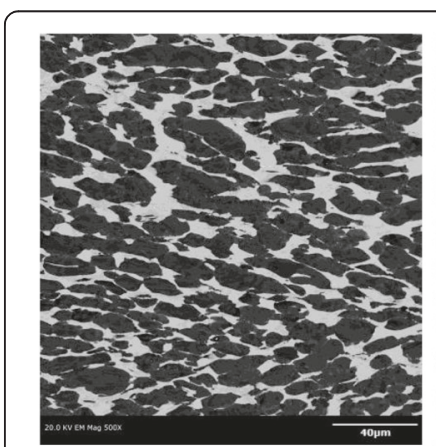

a

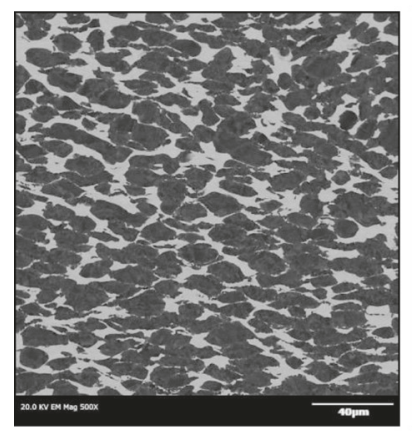

d

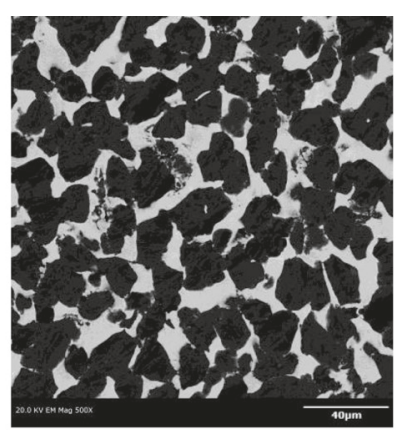

b

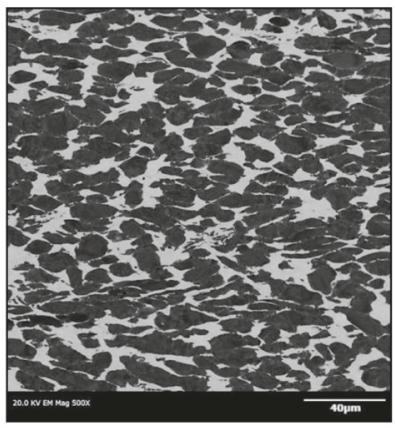

e

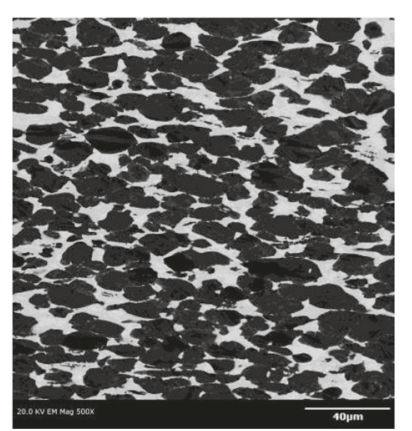

C

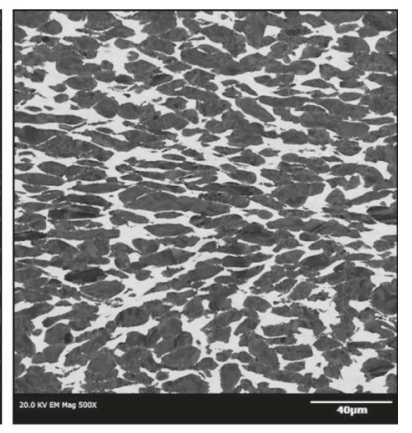

f

\section{$100 \mu \mathrm{m}$}

Fig. 7 Backscattered-electron micrographs of faces a 1, b 2, and $\mathbf{c} 3$ from the $899{ }^{\circ} \mathrm{C}$ axial-compression test and micrographs collected from face 1 in $\mathbf{d}$ axial, e radial, and $\mathbf{f} 45^{\circ}$ compression samples tested at $899^{\circ} \mathrm{C}$ 
Table 3 Summary of EBSD scans included in the dataset

\begin{tabular}{llllll}
\hline Dataset & \# rows & \# columns & Tile size $(\mu \mathrm{m})$ & Step size $(\mu \mathrm{m})$ & Overlap $(\mu \mathrm{m})$ \\
\hline Billet_Face1 & 23 & 70 & $260 \times 260$ & 5 & 0 \\
Billet_Face2 & 29 & 29 & $180 \times 180$ & 5 & 0 \\
Billet_Face3 & 40 & 40 & $180 \times 180$ & 5 & 0 \\
Forging_45_Face1 & 18 & 84 & $180 \times 180$ & 5 & 0 \\
Forging_45_Face2 & 45 & 45 & $180 \times 180$ & 5 & 10 \\
Forging_45_Face3 & 19 & 45 & $180 \times 180$ & 5 & 10 \\
Forging_Axial_Face1 & 19 & 89 & $180 \times 180$ & 5 & 10 \\
Forging_Axial_Face2 & 45 & 45 & $180 \times 180$ & 5 & 10 \\
Forging_Axial_Face3 & 19 & 45 & $180 \times 180$ & 5 & 10 \\
Forging_Radial_Face1 & 20 & 98 & $180 \times 180$ & 2.5 & 10 \\
Forging_Radial_Face2 & 45 & 45 & $180 \times 180$ & 5 & 10 \\
Forging_Radial_Face3 & 19 & 45 & $180 \times 180$ & 5 & - \\
Forging_Axial_HalfSample & - & - & - & 15 & - \\
Forging_45-FullSample & - & - & - & 15 &
\end{tabular}

scans performed on the as-received billet and the compression samples are illustrated in Figs. 4 and 5. It is noteworthy that the reference frame used for crystallographic orientations is not the same reference frame used to denote the spatial coordinates of the pixels [17] and that the orientation of the hexagonal alpha phase at the Euler angle $(0,0,0)$ has the

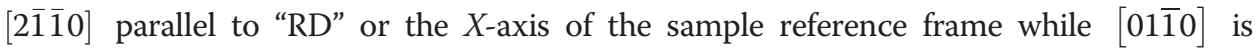
parallel to "TD" or the $Y$-axis. The same billet material was used for all of the compression experiments. Hence, it was assumed that the as-measured billet texture reasonably approximated the starting condition for each experiment since all compression samples were cut from the same radial position in the billet. As a result, it is necessary to rotate the morphological and crystallographic orientations of the MTRs for the $45^{\circ}$ and $90^{\circ}$ (radial) compression specimens prior to simulating the respective deformation experiments. Special attention should be paid to face 1 in the billet (Fig. 4) as it was physically oriented $90^{\circ}$ relative to face 3 when the data was collected.

If one is interested in assessing the global texture evolution in the final forgings by combining the measurements from the three mutually perpendicular faces, it is necessary to apply additional transformations to the post-compression datasets. The following rotation matrices, $R_{i}$, should be used to transform the as-collected orientation, $g$, to the new orientation, $g^{\prime}=R_{i} g$, which coincides with the face 1 reference frame.

$$
\begin{aligned}
& R_{2}=\left[\begin{array}{ccc}
0 & 0 & -1 \\
0 & 1 & 0 \\
1 & 0 & 0
\end{array}\right] \\
& R_{3}=\left[\begin{array}{ccc}
1 & 0 & 0 \\
0 & 0 & -1 \\
0 & 1 & 0
\end{array}\right]
\end{aligned}
$$

\section{Segmentation and quantification of microtexture}

The stitched, but otherwise raw, EBSD data were further processed for the purposes of feature identification and segmentation of MTRs. For this purpose, a new DREAM.3D 
(BlueQuartz Software, Springboro, OH) filter [18] was developed to overcome the shortcomings of current algorithms. Presently, most EBSD analysis software utilizes a so-called "burn" algorithm to group pixels of discrete measurements into grains (features) based on a user-defined misorientation criterion. The misorientation operator acts on only first-nearest-neighbor pixels and therefore requires contiguity of similarly oriented pixels to form a grain. Such an approach is incapable of properly segmenting MTRs, however, because adjacent alpha particles may not necessarily be interconnected, either in reality or due to the scan resolution. This is especially true after solution heat treatment high in the alpha+beta-phase field where primary-alpha particles are effectively insulated from one another by a matrix of transformed beta. There are rare instances in which the primary-alpha particles and adjacent secondary alpha adopt a similar orientation such that the classic burn algorithm can capture the MTRs; however, this is a rare exception. Despite an absence of connectivity, similarly oriented alpha particles tend to behave as a single microstructural unit as evidenced by observations of large, faceted initiation sites on dwell-fatigue fracture surfaces of alloys with low volume fractions of primary alpha. Such observations raise two important questions that impact the choice of segmentation parameters, which are founded in the context of material behavior, i.e.,: (i) at what distance do two alpha particles of the same orientation no longer interact with one another and (ii) how does this value change with misorientation and particle size? Therefore, in the present work and the work by Tucker et al. [19], a two-step feature identification process was used.

First, alpha particles are identified using the classical misorientation parameter with a relatively tight tolerance, $\sim 2^{\circ}$, similar to the $1.25^{\circ}$ used in the pioneering work of Woodfield et al. [4]. Second, the alpha particles are considered for grouping as MTRs in a burn algorithm that operates on the previously identified particles. The contiguity requirement is not enforced here; instead, the "burn" is performed within a userspecified search radius, which looks at centroid-to-centroid distances of neighboring alpha particles (so-called neighborhoods). The search radius is entered as a multiple of the average alpha-particle diameter, and alpha particles satisfying these criteria are grouped into MTRs based on a moderately wide $c$-axis misorientation tolerance, viz, $20^{\circ}$. The second-tier search proceeds until all previously identified particles are assigned to a parent MTR or left as an individual feature. The $c$-axis is used as opposed to the classic misorientation because the elastic and plastic response varies strongly with changes in $c$-axis orientation relative to the stress axis per se, whereas no or very minor changes are observed for rotations about [0001] at a constant orientation. Moreover, crack propagation occurs on or near basal planes [20] at small-crack lengths, so tilt boundaries offer resistance to crack propagation through deflection of the crack path and the development of roughness-induced closure. Basal twist boundaries, on the other hand, offer no such similar resistance to small fatigue-crack growth as evidenced by previous marker band studies [21].

It is noteworthy that this filter can be used within other pipelines in the DREAM.3D application suite and therefore has broad applicability to the study of "neighborhoodsensitive" properties in other materials. We have used a factor of five times the average particle size for this work which, due to the coarse resolution, was essentially the step size of the scan $(5 \times 5 \mu \mathrm{m}=25 \mu \mathrm{m}$ search radius). As illustrated in Fig. 8, the upper tail of the measured MTR size distribution, which is typically the region responsible for 


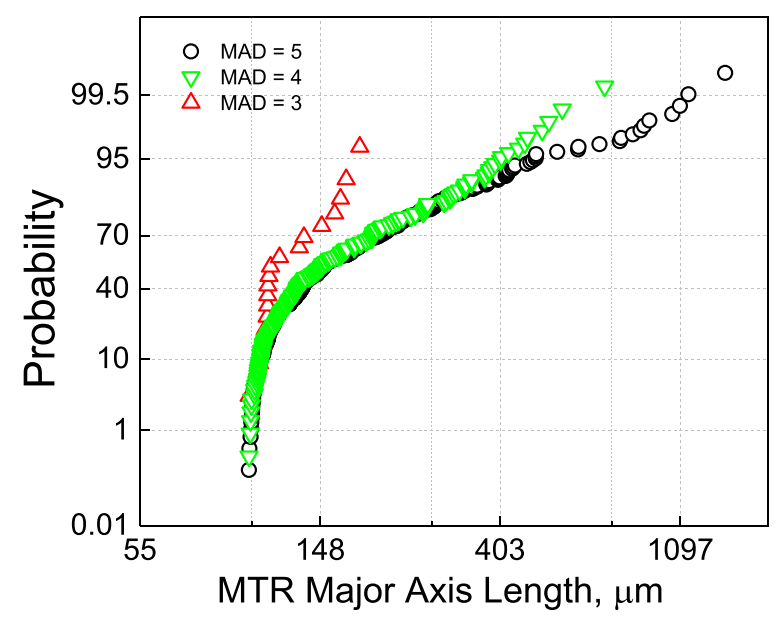

Fig. 8 Probability plot showing the sensitivity of the MTR major-axis length to the search-range parameter in terms of alpha-particle mean average diameter (MAD). The data are shown as a three-parameter lognormal distribution with a major-axis threshold of $100 \mu \mathrm{m}$

life-limiting dwell-fatigue failures, is quite sensitive to the search-radius parameter, which, at present, does not have a physical basis for its specification. Saint-Venant's principle may serve as a working hypothesis, which indicates that the influence of a given object ceases at approximately three times the feature size. For this analysis, we have used a set of empirically determined segmentation parameters, and thus, we have also provided the raw data and the associated pipeline to process the data using DREAM.3D such that the reader can test other segmentation parameters. Furthermore, all identified features with semi-major (ellipse fit) axes less than $100 \mu \mathrm{m}$, corresponding to approximately seven average alpha particles, have been thresholded to define the lower-bound dimension of the MTR. For other purposes, the threshold value may need to be considerably larger depending on the problem of interest.

Example crystal orientation maps and the corresponding segmented MTRs (colored by equivalent diameter) are shown in Figs. 9 and 10. The size and shape of the MTRs in each two-dimensional plane were approximated as ellipses quantified using the

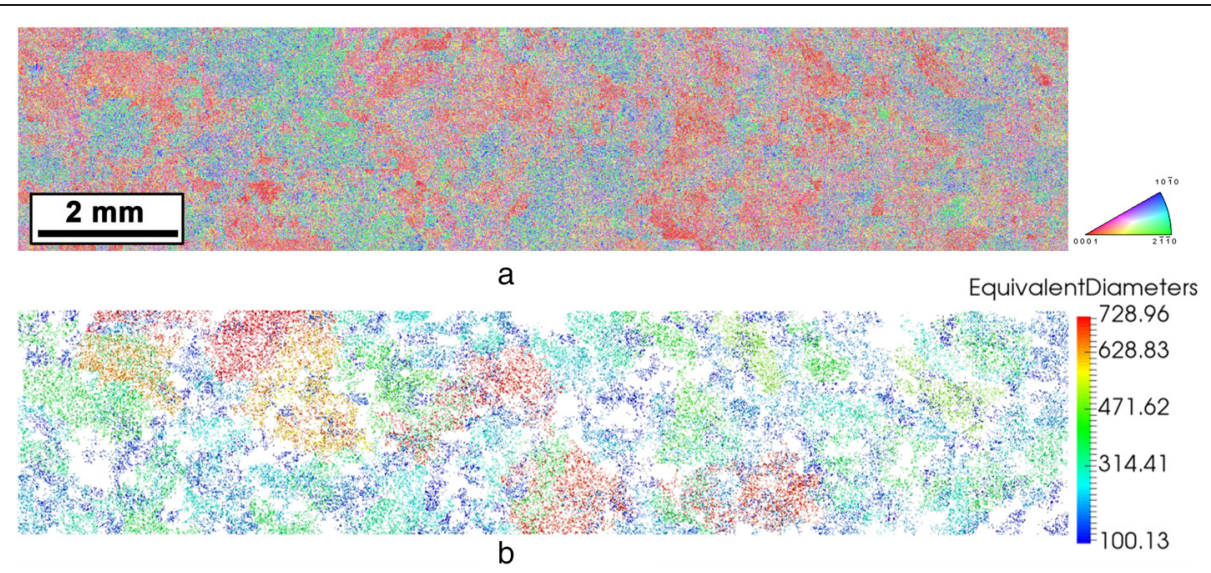

Fig. 9 a Radial-direction (normal to plane of scan) inverse-pole-figure map for face 1 of the $899^{\circ} \mathrm{C}$ axial-compression sample and $\mathbf{b}$ segmented MTRs colored by circle equivalent diameter in micrometers 


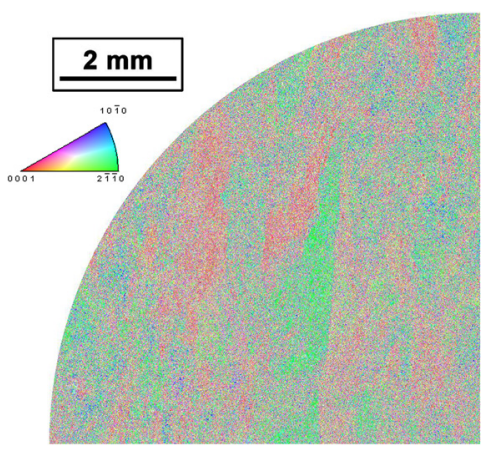

a

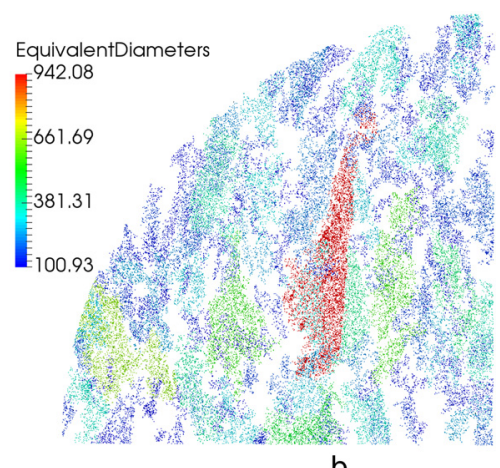

$\mathrm{b}$

Fig. 10 a Forging-direction (normal to plane of scan) inverse-pole-figure map of face 2 of the $899^{\circ} \mathrm{C}$ radial compression sample and $\mathbf{b}$ segmented MTRs colored by circle equivalent diameter in micrometers

length of their major and minor axes and the inclination of the major axis with respect to the billet or forging direction. Table 4 shows basic statistics for the MTR sizes on face 1 of the $45^{\circ}$ compression sample. The statistics were applied to independent quartiles of the data based on the MTR major-axis lengths. Figures 11 and 12 depict the effect of strain on the evolution of major and minor MTR axis lengths as a function of MTR aspect ratio and inclination angle for face 1 in the billet and following hot compression in the $45^{\circ}$ direction. The aspect ratio is defined as the major/minor axis lengths while the inclination angle reported is relative to a direction perpendicular to the compression axis. Figure 11a shows that the great majority ( $80 \%)$ of the MTRs have aspect ratios less than $\sim 5$. The MTR major-axis inclination angle data in Fig. 11b has been rotated into the same reference frame as the $45^{\circ}$ compression sample, and

Table 4 General statistics for face 1 of the $45^{\circ}$ compression sample separated into quartiles based on MTR major axis

\begin{tabular}{|c|c|c|c|c|c|c|}
\hline Quartile & Metric & Aspect Ratio & Major Axis, $\mu m$ & Minor Axis, $\mu \mathrm{m}$ & $\begin{array}{l}\text { Equivalent } \\
\text { Diameter, } \mu \mathrm{m}\end{array}$ & $\begin{array}{l}\text { Major Axis } \\
\text { Inclination, deg }\end{array}$ \\
\hline \multirow[t]{4}{*}{ Fourth (75-100\%) } & Mean & 5.6 & 343.0 & 67.2 & 175.1 & 5.0 \\
\hline & Std Dev & 2.2 & 158.4 & 30.1 & 71.8 & 4.5 \\
\hline & Min & 1.9 & 205.2 & 21.3 & 89.0 & 0.0 \\
\hline & Max & 13.3 & 1062.4 & 202.3 & 475.7 & 24.6 \\
\hline \multirow[t]{4}{*}{ Third (50-75\%) } & Mean & 4.1 & 169.6 & 46.8 & 100.5 & 6.9 \\
\hline & Std Dev & 1.6 & 19.7 & 18.3 & 21.3 & 6.3 \\
\hline & Min & 1.3 & 142.5 & 17.1 & 66.5 & 0.0 \\
\hline & Max & 11.3 & 205.0 & 126.9 & 210.1 & 36.8 \\
\hline \multirow[t]{4}{*}{ Second (25-50\%) } & Mean & 3.6 & 128.7 & 40.4 & 80.6 & 8.6 \\
\hline & Std Dev & 1.3 & 7.6 & 16.0 & 14.8 & 9.4 \\
\hline & Min & 1.2 & 116.9 & 14.4 & 46.5 & 0.0 \\
\hline & Max & 8.4 & 142.3 & 108.8 & 135.5 & 89.1 \\
\hline \multirow[t]{4}{*}{ First (0-25\%) } & Mean & 3.4 & 107.8 & 36.1 & 69.3 & 9.8 \\
\hline & Std Dev & 1.3 & 4.8 & 14.4 & 12.4 & 12.0 \\
\hline & Min & 1.1 & 100.3 & 14.5 & 41.8 & 0.0 \\
\hline & Max & 7.0 & 116.9 & 96.0 & 111.1 & 85.0 \\
\hline
\end{tabular}




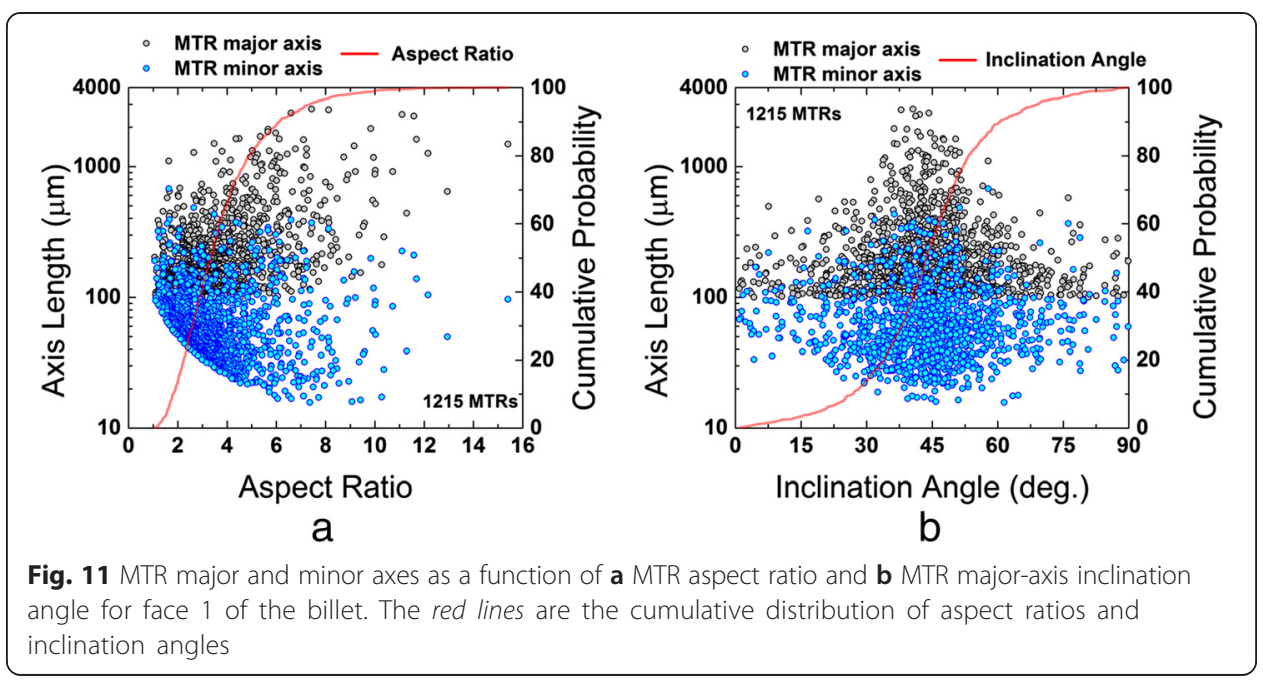

hence, the initial billet axis is located at $45^{\circ}$. Following compression at $45^{\circ}$ to the billet axis (Fig. 12), one can see that the major axes of most MTRs were rotated nearly perpendicular to the compression direction and were reduced in size. The MTR aspect ratios were only moderately impacted, exhibiting a slight increase, as indicated by the similar shape and position of the cumulative distributions. The Excel $^{\mathrm{mt}}$ file in the MTR processing folder contains this information for all datasets.

Crystal orientation maps of the full cross-section of the $45^{\circ}$ and half cross-section of the axial-compression samples are shown in Figs. 13 and 14, respectively. These datasets show the interaction of the MTRs with the continuum-scale metal flow. For instance, Fig. 13 shows the retention of larger MTRs in the dead metal zone while there is clear bending and distortion of the MTRs in Fig. 14.

\section{Potential applications of the dataset}

As mentioned in the introduction, there have been a number of advances in modeling microstructure evolution during TMP of titanium alloy over the past two decades. In

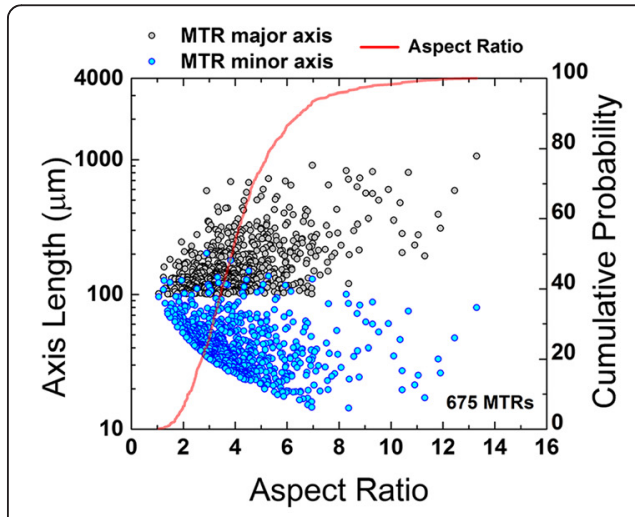

a

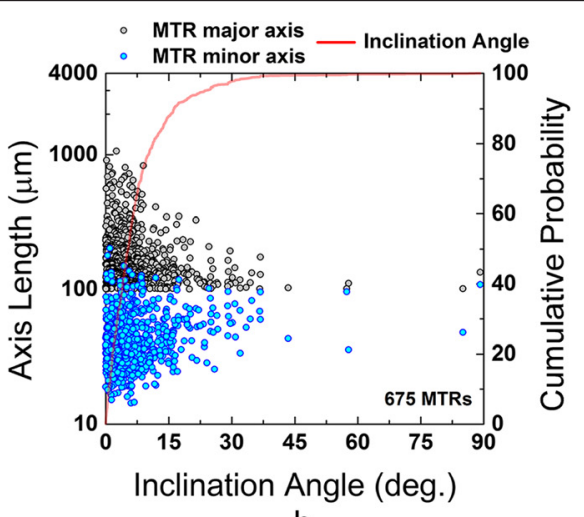

b

Fig. 12 MTR major and minor axes as a function of a MTR aspect ratio and $\mathbf{b}$ MTR major-axis inclination angle for face 1 of the $45^{\circ}$ compression sample. The red lines are the cumulative distribution of aspect ratios and inclination angles 


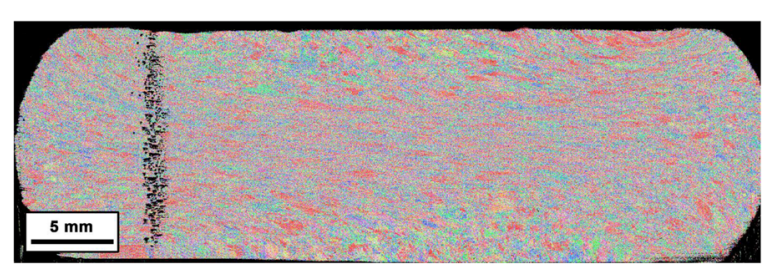

Fig. 13 Normal direction inverse-pole figure map of the entire cross-section of the $899^{\circ} \mathrm{C} 45^{\circ}$ compression sample. The black vertical line on the left is an electropolishing artifact

this regard, the present dataset attempts to fill an important gap in the current knowledge base, namely, quantifying and modeling how the volume fraction, size, and shape of MTRs evolve during TMP. In addition to this primary goal, the dataset may also serve as a benchmark for comparing/contrasting methods for characterizing MTRs developed in other laboratories or for performing sensitivity analyses on MTR segmentation methods. While the principal focus in the present work was on MTRs, the dataset also contains images of the primary-alpha particles in the as-received billet and upset forged cylinders, thereby providing information to characterize the details of microstructure evolution at a finer length scale. These images were segmented to identify the alpha- and beta-phase volume fractions as this is a necessary parameter in strain-partitioning calculations in self-consistent crystal plasticity models. The volume fraction of alpha was quite similar over the range of fields of view considered and over the various imaging planes with the average measurement being $~ 71 \%$.

One of the most attractive features of the present dataset concerns the fact that it was extracted from carefully designed experiments intended to permit simplifying certain assumptions. First, the compression tests were performed isothermally at a relatively low strain rate $\left(0.01 \mathrm{~s}^{-1}\right)$ to minimize changes in flow stress due to temperature changes associated with die chill and deformation heating as well as grossly non-uniform flow associated with flow localization and shear band formation. Second, the region characterized with electron microscopy was at the center of the sample which experienced nearly perfect homogeneous, axisymmetric compression. This simple state of deformation enables researchers without finite-element codes to

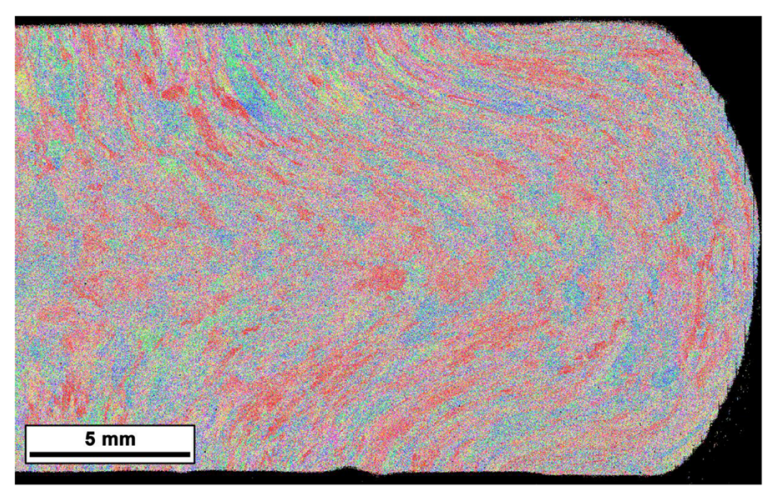

Fig. 14 Normal direction inverse map of the $899^{\circ} \mathrm{C}$ axial-compression sample 
perform relevant crystal-plasticity simulations using the simple (normalized) velocity gradient, $L$ :

$$
L=\left[\begin{array}{ccc}
0.5 & 0 & 0 \\
0 & 0.5 & 0 \\
0 & 0 & -1
\end{array}\right]
$$

On the other hand, those with coupled or uncoupled crystal plasticity finite-element (CPFE) models can take advantage of the two large scans on face 1 of the axial and $45^{\circ}$ compression samples. As illustrated in Fig. 14, continuum-scale metal flow patterns contribute significantly to the evolution of MTRs during forging. Such non-axisymmetric deformation paths require rigid-body rotations due to the metal flow being considered within the crystal plasticity framework, and thus, these two datasets are provided for this type of detailed validation on at least one plane.

The dataset can also be used to develop improved shape/orientation descriptors for MTRs. The initial choice of a simple size/shape descriptor is related to ongoing efforts to account for the role of MTRs in a linear-elastic-fracture-mechanics framework for service failure. In particular, X-ray computed tomography [20] and small-crack-growth [10] studies have indicated that cracks may grow rapidly and consume the first MTR in which they initiate before propagating into the surrounding material. For this reason, the cracked MTR is approximated as an ellipse in the plane perpendicular to the stress axis for the purpose of calculating the stress intensity range, $\Delta \mathrm{K}$, which provides the driving force for continued long crack growth. In the future, refined analyses will certainly need to take into account a more precise representation of the MTR shape and boundary character.

Finally, the dataset can be used to study the internal structure and distribution of orientations within the MTRs and how this factor evolves with strain and crystallographic orientation. These internal misorientations are an important feature that may impact local crack growth rates within individual MTRs. It is noteworthy, however, that preliminary efforts to quantify MTR "intensity" suggest that this variable is strongly dependent on EBSD scan resolution. Thus, it would be interesting to compare segmentation parameters on the radial sample face 2 dataset $(2.5 \mu \mathrm{m}$ resolution) in the as-collected and once-coarsened states.

\section{Summary}

A dataset comprising isothermal hot-compression test data, microstructural characterization before and after compression, and FEM simulations of the compression experiment has been summarized for the primary purpose of providing important experimental details for developing and validating robust models to describe the size and shape evolution of MTRs during TMP. While we have chosen a relatively simple mode of deformation (analogous to pancake forging), the evolution of crystallographic orientations and grain shape in plastically anisotropic materials during complex, largestrain metalworking operations is of general interest and has broad applicability.

\section{Availability and requirements of software used}

Project name: Microsoft Excel

Project home page: https://products.office.com/en-us/excel

Operating system(s): platform independent 
Programming language: $\mathrm{C} / \mathrm{C}++$

Other requirements: $\mathrm{n} / \mathrm{a}$

License: EULA

Project name: DEFORM ${ }^{\mathrm{Tm}}$

Project home page: http://www.deform.com

Operating system(s): platform independent

Programming language: Fortran

Other requirements: $\mathrm{n} / \mathrm{a}$

License: EULA

Project name: Orientation Imaging Microscopy Analysis

Project home page: http://www.edax.com/Products/EBSD/OIM-Data-Analysis-

Microstructure-Analysis.aspx

Operating system(s): platform independent

Programming language: unknown

Other requirements: $\mathrm{n} / \mathrm{a}$

License: EULA

Project name: DREAM.3D

Project home page: http://dream3d.bluequartz.net/

Operating system(s): platform independent

Programming language: $\mathrm{C}++$

Other requirements: none for pre-compiled binaries

Other requirements (to compile from source): Git, CMake, Doxygen, HDF5, Boost,

Eigen, Intel Threading Building Blocks, Qt 5.4.x, Qwt,

License: BSD

Project name: MATLAB

Project home page: http://www.mathworks.com

Operating system(s): platform independent

Programming language: C, Java, Fortran, LAPACK, ARPACK and MATLAB (.m files)

Other requirements: $\mathrm{n} / \mathrm{a}$

License: EULA

Acknowledgements

This work was performed as part of the in-house research activities of the Air Force Research Laboratory's

Materials and Manufacturing Directorate. The support of laboratory management is greatly appreciated. Several

of the authors (JS, JT, SS, PF) were supported through Air Force contract FA8650-10-D-5226 during the time

this work was completed.

\section{Authors' contributions}

ALP conceived the study, collected the EBSD data, coordinated the efforts at AFRL, and drafted the manuscript; ALP and JCT devised the microtexture segmentation routine while JCT implemented the algorithms in DREAM.3D; JS collected the BSE images, performed the MTR segmentation, and coordinated uploading the dataset to the NIST server; PNF performed and reduced the data from the isothermal compression tests; SS developed and validated the finite-element models; and SLS helped design the experiments, assisted with data analysis, and also assisted with drafting the manuscript. All authors read and approved the final manuscript. 


\section{Author details}

${ }^{1}$ Air Force Research Laboratory, AFRL/RXCM, Wright-Patterson AFB, OH 45433, USA. ²UES Inc., 4401 Dayton-Xenia Rd, Beavercreek, OH 45432, USA. ${ }^{3}$ Present address: Exponent, Inc., 3350 Peachtree Rd NE \#1125, Atlanta, GA 30326, USA. ${ }^{4}$ Srivatsa Consulting, LLC, Cincinnati, OH 45249, USA.

Received: 14 April 2016 Accepted: 10 August 2016

Published online: 15 September 2016

\section{References}

1. Van Stone RH, Low JR Jr, Shannon JL Jr (1978) Investigation of the fracture mechanism of Ti-5Al-2.5Sn at cryogenic temperatures. Metall Trans A 9:539-47

2. Bowen AW (1975) Influence of crystallographic orientation on fatigue crack growth in strongly textured Ti-6Al-4V. Acta Metall 23(11):1401-1409

3. Evans WJ, Jones JP, Whittaker MT (2005) Texture effects under tension and torsion loading conditions in titanium alloys. Int J Fatigue 27:1244-1250

4. Woodfield AP, Gorman MD, Corderman RR, Sutliff JA, Yamrom B (1996) Effect of microstructure on dwell fatigue behavior of Ti-6242. Titanium'95: Science and Technology, P.A. Blenkinsop, W.J. Evans, and H.M. Flower, eds., Institute of Materials, Birmingham, UK:1116-23

5. Toubal L, Bocher P, Moreau A, Levesque D (2010) Macroregion size measurements in bimodal titanium forgings using two-dimensional autocorrelation method. Metall Mater Trans A 41:744-750

6. Bridier F, Villechaise P, Mendez J (2005) Analysis of the different slip systems activated by tension in a $a / \beta$ titanium alloy in relation with local crystallographic orientation. Acta Mater 53:555-567

7. Echlin M, Stinville JC, Miller VM, Lenthe WC, Pollock TM (2016) Incipient slip and long range plastic strain localization in microtextured Ti-6Al-4V titanium. Acta Mater 114:164-175.z

8. Semiatin SL, Furrer DU, Modeling of microstructure evolution during the thermomechanical processing of titanium alloys, ASM Handbook, Vol. 22A, Tenth Edition, ASM International, Materials Park, OH, 2009, p. 522

9. Semiatin SL, Shevchenko SV, Ivasishin OM, Glavicic MG, Chun YB, and Hwang SK, Modeling and simulation of texture evolution during the thermomechanical processing of titanium alloys, ASM Handbook, Vol. 22A, Tenth Edition, ASM International, Materials Park, OH, 2009, p. 536

10. Pilchak AL, Hutson A, Porter WJ, Buchanan DJ and John R (2016) Growth of small and long fatigue cracks in Ti-6Al-4V subjected to cyclic and dwell fatigue, Proc. of the $13^{\text {th }}$ World Conference on Titanium, ed. V. Venkatesh et al. (Warrendale, PA: The Minerals, Metals \& Materials Society; Hoboken, NJ: John Wiley \& Sons, 2016), pp. 993-998.

11. Pilchak AL (2014) A simple model to account for the role of microtexture on fatigue and dwell fatigue lifetimes of titanium alloys. Scr Mater 74:68-71

12. Dataset archived on the NIST repository: http://hdl.handle.net/11256/647

13. Pilchak AL, Szczepanski CJ, Shaffer JA, Salem AA, Semiatin SL (2013) Characterization of microstructure, texture and microtexture in near-alpha titanium mill products. Metall Mater Trans A 44:4881-4890

14. Altan T (1983) in Metals forming: fundamentals and applications, ASM International, Materials Park, OH, Chapter 10

15. Shiveley AR, Shade PA, Pilchak AL, Tiley JS, Kerns R (2011) A novel method for acquiring large scale automated scanning electron microscopy data. J Microsc 244(2):181-186

16. Pilchak AL, Shiveley AR, Tiley JS, Ballard DL (2011) AnyStitch: a tool for combining electron backscatter diffraction data sets. J Microsc 244(1):38-44

17. Pilchak AL, Shiveley AR, Shade PA, Tiley JS, Ballard DL (2012) Using cross-correlation for automated stitching of two-dimensional multi-tile electron backscatter diffraction data. J Microsc 248(2):172-186

18. Groeber MA, Jackson MA (2014) DREAM.3D: a Digital Representation Environment for the Analysis of Microstructure in 3D. Integrating Materials and Manufacturing Innovation 3(5):1-17

19. Tucker JC, Pilchak AL, Groeber MA, Semiatin L (2015) Objective characterization, synthetic building and targeted analysis of microtextured regions from electron backscatter diffraction data. The 13th World Conference on Titanium 2015, San Diego, CA, August 2015

20. Pilchak AL, Williams JC (2011) Observations of facet formation in near-a titanium and comments on the role of hydrogen. Metall Mater Trans A 42(4):1000-1027

21. Pilchak AL, Bhattacharjee A, Rosenberger AH, Williams JC (2009) Low $\Delta K$ faceted crack growth in titanium alloys. Int J Fatigue 31:989-994

\section{Submit your manuscript to a SpringerOpen ${ }^{\circ}$ journal and benefit from:}

- Convenient online submission

- Rigorous peer review

Immediate publication on acceptance

- Open access: articles freely available online

- High visibility within the field

- Retaining the copyright to your article 